\title{
Shape induced segregation and anomalous particle transport under spherical confinement $\odot$
}

Cite as: Phys. Fluids 32, 053307 (2020); https://doi.org/10.1063/5.0002906

Submitted: 28 January 2020 . Accepted: 15 April 2020 . Published Online: 08 May 2020

Abhinendra Singh (D), Jiyuan Li (李冀圆) (D), Xikai Jiang (蒋胥恺) (D), Juan P. Hernández-Ortiz (D), Heinrich M. Jaeger, and Juan J. de Pablo (i)

\section{COLLECTIONS}

F This paper was selected as Featured
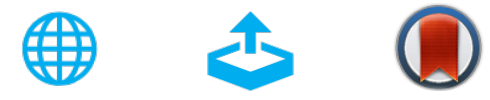

\section{ARTICLES YOU MAY BE INTERESTED IN}

Asymmetric flows of complex fluids past confined cylinders: A comprehensive numerical study with experimental validation

Physics of Fluids 32, 053103 (2020); https://doi.org/10.1063/5.0008783

Structure and dynamics of hydrodynamically interacting finite-size Brownian particles in a spherical cavity: Spheres and cylinders

The Journal of Chemical Physics 152, 204109 (2020); https://doi.org/10.1063/1.5139431

\section{Gravel packing: How does it work?}

Physics of Fluids 32, 053308 (2020); https://doi.org/10.1063/5.0001607

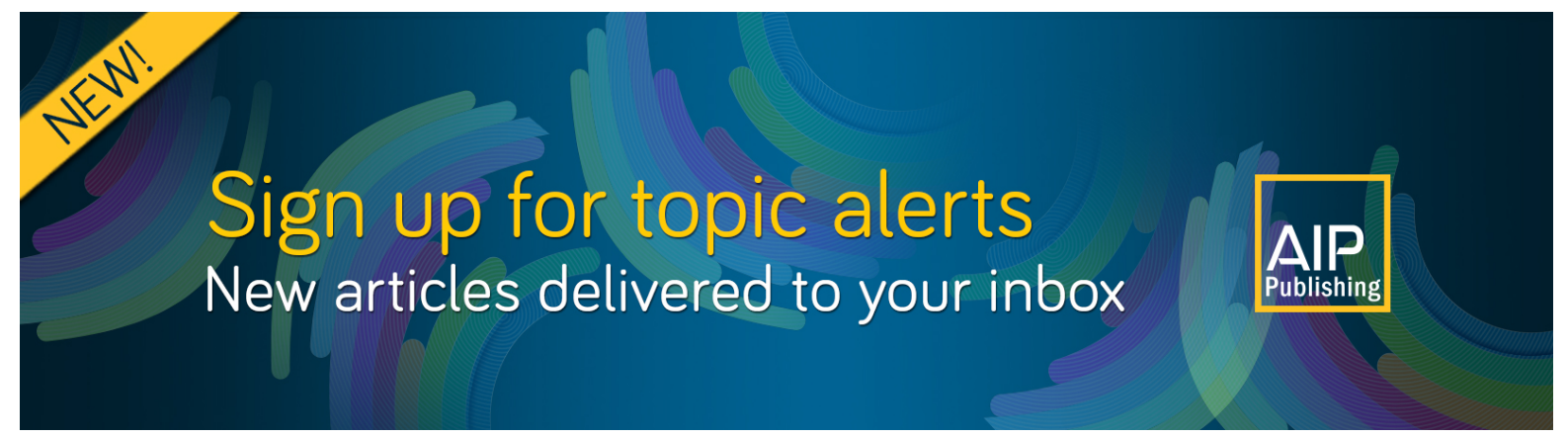




\title{
Shape induced segregation and anomalous particle transport under spherical confinement
}

\author{
Cite as: Phys. Fluids 32, 053307 (2020); doi: 10.1063/5.0002906 \\ Submitted: 28 January 2020 - Accepted: 15 April 2020 • \\ Published Online: 8 May 2020
}

\begin{abstract}
Abhinendra Singh, ${ }^{1,2}$ (D) Jiyuan Li (李冀圆), (D) Xikai Jiang (蒋笚恺), ${ }^{1,3}$ (D) Juan P. Hernández-Ortiz, Heinrich M. Jaeger, ${ }^{2,6}$ and Juan J. de Pablo ${ }^{1,7, b)}$ (D)
\end{abstract}

\begin{abstract}
AFFILIATIONS
${ }^{1}$ Pritzker School of Molecular Engineering, University of Chicago, Chicago, Illinois 60637, USA

2James Franck Institute, University of Chicago, Chicago, Illinois 60637, USA

${ }^{3}$ State Key Laboratory of Nonlinear Mechanics, Institute of Mechanics, Chinese Academy of Sciences, Beijing 100190, China

${ }^{4}$ Department of Materials and Nanotechnology, Universidad Nacional de Colombia-Medellín, Medellín 050034, Colombia

${ }^{5}$ Colombia/Wisconsin One-Health Consortium, Universidad Nacional de Colombia-Medellín, Medellín 050034, Colombia

${ }^{6}$ Department of Physics, The University of Chicago, Chicago, Illinois 60637, USA

${ }^{7}$ Materials Science Division, Argonne National Laboratory, Lemont, Illinois 60439, USA
\end{abstract}

a) Electronic mail: jphernandezo@unal.edu.co

${ }^{b}$ Author to whom correspondence should be addressed: depablo@uchicago.edu

\begin{abstract}
Colloidal or nanoparticle mobility under confinement is of central importance for a wide range of physical and biological processes. Here, we introduce a minimal model of particles in a hydrodynamic continuum to examine how particle shape and concentration affect the transport of particles in spherical confinement. Specifically, an immersed boundary-general geometry Ewald-like approach is adopted to simulate the dynamics of spheres and cylinders under the influence of short- and long-range fluctuating hydrodynamic interactions with appropriate non-slip conditions at the confining walls. An efficient $O(N)$ parallel finite element algorithm is used, thereby allowing simulations at high concentrations, while a Chebyshev polynomial approximation is implemented in order to satisfy the fluctuation-dissipation theorem. A concentration-dependent anomalous diffusion is observed for suspended particles. It is found that introducing cylinders in a background of spheres, i.e., particles with a simple degree of anisotropy, has a pronounced influence on the structure and dynamics of the particles. First, increasing the fraction of cylinders induces a particle segregation effect, where spheres are pushed toward the wall and cylinders remain near the center of the cavity. This segregation leads to a lower mobility for the spheres relative to that encountered in a system of pure spheres at the same volume fraction. Second, the diffusive-to-anomalous transition and the degree of anomaly quantified by the power law exponent in the mean square displacement vs time relation both increase as the fraction of cylinders becomes larger. These findings are of relevance for studies of diffusion in the cytoplasm, where proteins exhibit a distribution of size and shapes that could lead to some of the effects identified in the simulations reported here.
\end{abstract}

Published under license by AIP Publishing. https://doi.org/10.1063/5.0002906

\section{INTRODUCTION}

Colloidal and nanoparticle diffusion in confined environments arises in a wide range of scientific and engineering systems, including living cells, mesoporous materials, and microfluidic devices. ${ }^{1-6}$ It is also of interest for energy generation processes that rely on salinity or electrostatic gradients in pores. ${ }^{7-10}$ In the particular case of the cytoplasm, the diffusion of biomolecules underpins a variety of intracellular metabolic, translational, and locomotion processes, to name a few. ${ }^{11-16}$ Interestingly, particle diffusion in these confined systems is often found to be severely hindered and anomalous. ${ }^{17-19}$ The mechanisms behind those observations, however, remain poorly understood.

Several studies have examined particle mobility in living cells ${ }^{20,21}$ by relying on Brownian dynamics (BD) simulations. In such studies, biological macromolecules have been represented as 
spheres, and numerical simulations have found evidence of hindered diffusion, in agreement with experimental results. Majority of previous studies, however, have failed to consider hydrodynamic interactions (HIs) between particles or between particles and the confining walls, and the presence of boundaries has shown to affect the particle mobility. ${ }^{22,23}$ Some exceptions are provided by the work of Ando and Skolnick ${ }^{24}$ and Chow and Skolnick, ${ }^{25}$ who included hydrodynamic interactions between particles but did not enforce the no-slip boundary condition at the walls. More recently, Stokesian dynamics (SD) simulations of spheres by Aponte-Rivera et al. ${ }^{26,27}$ considered both far- and near-field (lubrication) hydrodynamic interactions (HIs) between particles and walls. The authors demonstrated that HIs have a pronounced influence on the local structure and the short-time and long-time diffusive behavior of particle suspensions. The framework employed by these authors relied on SD and was restricted to a homogeneous system of spherical particles. $^{2}$

Recently, we have introduced an efficient computational approach in order to overcome some of the limitations of other available numerical approaches for hydrodynamic interactions. In particular, this approach can be easily extended to particles of arbitrary shape dispersed in a confined geometry also of arbitrary shape. ${ }^{28-30}$ An Immersed-Boundary (IB) method is used to represent the suspended finite-sized particles. A parallel Finite ElementGeneral geometry Ewald-like method (pFE-GgEm) ${ }^{28}$ is used to calculate the confined Green's functions, which relies on a Chebyshev polynomial approximation to satisfy the fluctuation-dissipation theorem. In our recent work, ${ }^{30}$ we relied on this approach to compare the structure of pure spherical and pure cylindrical particles confined in a spherical cavity. It was found that cylindrical particles diffuse slower as compared to spherical particles of the same volume and at the same volume fraction and that for cylinders, the transition from the diffusive to the sub-diffusive regime occurs at a lower volume fraction.

The studies mentioned above focused on pure spheres or cylinders confined in a spherical cavity. The more relevant case of mixtures of spheres and cylinders was not considered. Indeed, in applications (e.g., cytoplasm or colloidal suspensions), one rarely deals with systems of pure mono-disperse spheres, and it is, therefore, of interest to consider how mixtures behave relative to their pure counterparts. Note that limited experimental evidence with mixtures of particles of different sizes and shapes indicates that cells exhibit preferential accumulation of some particles near the nucleus. ${ }^{31,32}$ In those cases, size-based segregation was explained on the basis of a "sieving effect" that has been advanced in the dry granular segregation literature. ${ }^{33,34}$ An explanation for shape-based segregation was not proposed in that work. Other experimental studies, including a study of centrifugation of colloidal rods and spheres ${ }^{35}$ and a study in which milli-meter sized glass beads and rods were subject to strong vibration, ${ }^{36}$ have also reported segregation effects based on particle shape and proposed that hydrodynamic forces based on the different shapes contribute to that segregation.

Our particular goal here is to provide a standard against which past and future observations of segregation and diffusion can be compared by simulating mixtures of particles of equal volume but having a spherical or a cylindrical aspect ratio $h_{c} / r_{c}=2$. By doing so, we seek to rationalize past reports with new evidence for size-based segregation and mobility gradients in systems where dimensions and interactions are perfectly controlled, thereby eliminating or avoiding some of the complexity that arises in laboratory experiments. The outline of this paper is as follows: in Sec. II, we describe our numerical setup and methodology. Our results on the structure and dynamics of mixtures of spheres and cylinders are presented in Sec. III. We conclude the manuscript with a discussion of our findings in Sec. IV, along with a possible outlook for future studies.

\section{MODEL AND METHOD}

The system considered here consists of $N$ semi-rigid particles embedded in a viscous fluid of viscosity $\eta$ that are enclosed in a spherical cavity of radius $R$. The equations of motion under the condition of zero Reynolds number and zero Stokes number are given by

$$
\mathbf{F}^{H}+\mathbf{F}^{B}+\mathbf{F}^{C}+\mathbf{F}^{E V}+\mathbf{F}^{\text {ext }}=0,
$$

where $\mathbf{F}^{H}$ is the $6 N$ vector containing the hydrodynamic force/torque, $\mathbf{F}^{B}$ is the Brownian force/torque, $\mathbf{F}^{C}$ is the force/torque containing configuration terms, $\mathbf{F}^{E V}$ represents force/torque excluded volume contributions, and $\mathbf{F}^{\text {ext }}$ includes any external force/torque.

Evolution of the suspended particles, using Eq. (1), is achieved using the grand mobility or resistance tensors that relate the hydrodynamic force/torque with the translational and rotational velocities of the particles. ${ }^{37-39}$ Approaches such as $\mathrm{SD}^{40-46}$ and boundary integral methods (BIMs) ${ }^{37,47}$ are used extensively to solve the "mobility problem." The regularized Stokeslets, ${ }^{48}$ the accelerated BIM, ${ }^{49}$ and the Immersed Boundary (IB) ${ }^{44,50-53}$ provide examples of numerical methods developed to improve computational efficiency by simplifying or avoiding the calculation of the single- and double-layer hydrodynamic potentials of suspended particles. On the case of the Immersed Boundary (IB) approach, the surfaces of the suspended solids are represented by a distribution of discrete force densities on a surface mesh ( $N_{\text {IB }}$ immersed boundary nodes) that, together with a surface force description and Stokes equations, leads to the evolution of the suspended particles. The above mentioned approach is used in this study.

As in the boundary element method, ${ }^{47}$ in our simulations, the surface of each particle is discretized into a set of $N_{\text {IB }}$ nodes constituting a mesh (see Fig. 1). In order to maintain particle shape, volume, and surface, we define a spring potential on the surface nodes. Next, the force balance on each particle translates into $N_{\mathrm{IB}}$ surface nodes as follows:

$$
\mathbf{f}_{v}^{H}+\mathbf{f}_{v}^{B}+\mathbf{f}_{v}^{C}+\mathbf{f}_{v}^{E V}=\mathbf{0}
$$

for every node $v=1, \ldots, N_{\mathrm{IB}}$, where $\mathbf{f}_{v}^{H}$ is the hydrodynamic force, $\mathbf{f}_{v}^{B}$ is the Brownian force, $\mathbf{f}_{v}^{C}$ is the constitutive force, and $\mathbf{f}_{v}^{E V}$ is the force from all excluded volume interactions, including particle-particle and particle-wall.

The probability distribution function for the surface mesh positions in a Lagrangian frame of reference evolves according to a convection-diffusion equation of the Fokker-Planck type. ${ }^{4}$ We assume a continuous probability density and use the ChapmanKolmogorov equation white noise to obtain an equivalent stochastic differential equation for the motion of the mesh points, 


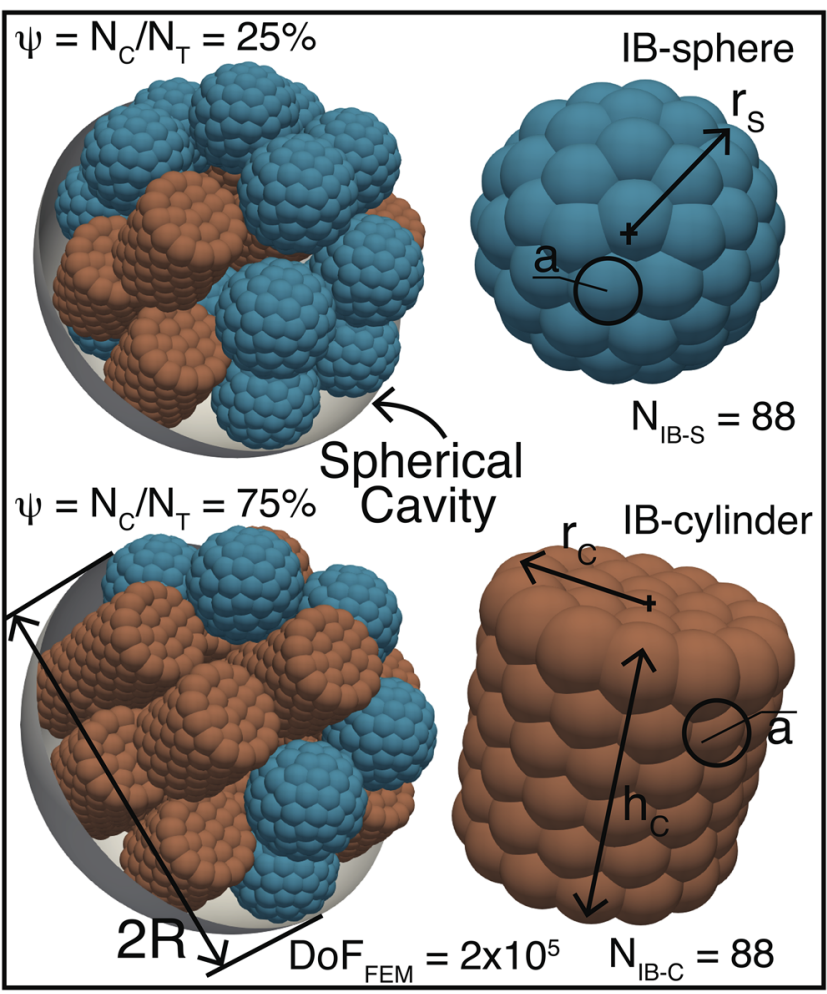

FIG. 1. Snapshots of the spherical cavity of radius $R$ containing spherical and cylindrical particles with $\phi_{H}=0.2$ for fraction of cylinders $\psi=N_{C} / N_{T}$ being 0.25 and 0.75 . The spherical particles radius is $r_{S}$, while the size of the cylindrical particles is determined by $r_{C}$ and $h_{C}$. The surface of the particles is given by a collection of discrete nodes that are connected to six neighbors, similar to boundary element discretizations, and with a characteristic node separation of $a \sim h \sim \xi_{\mathrm{BB}}^{-1}$. A repulsive Lennard-Jones excluded volume is included on each surface node, shown schematically in the particles' cross section by the black circles. The characteristic size of the repulsion is given by $\sigma=2.2 a$.

$$
d \mathbf{R}=\left[\mathbf{U}_{\mathbf{0}}+\mathbf{M} \cdot \mathbf{F}+\frac{\partial}{\partial \mathbf{R}} \cdot \mathbf{D}\right] d t+\sqrt{2} \mathbf{B} \cdot d \mathbf{W}
$$

where $\mathbf{U}_{0}$ denotes a $3\left(N \times N_{\mathrm{IB}}\right)$ vector of the unperturbed fluid velocity generated by external pressure differences or shear at the mesh point positions; $\mathbf{M}$ is the mobility tensor that includes Stokes' drag and the pair-wise Stokeslets accounting for the hydrodynamic interactions between mesh points; $\mathbf{D}=k_{B} T \mathbf{M}$ is the $\left(3 N \times N_{\mathrm{IB}}\right) \times(3 N$ $\left.\times N_{\text {IB }}\right)$ diffusion tensor; $\mathbf{F}$ is a $3\left(N \times N_{\text {IB }}\right)$ vector of the nonBrownian and non-hydrodynamic forces; $k_{B}$ is the Boltzmann constant; $T$ is the temperature; $\mathbf{M} \cdot \mathbf{F}$ is a convection term that represents the bead velocities arising from hydrodynamic interactions; the divergence of the diffusion tensor, $\frac{\partial}{\partial \mathbf{R}} \cdot \mathbf{D}$, is the first diffusive term resulting from the configuration-dependent mobility of the confined mesh points; $d \mathbf{W}$ is a random vector, the components of which are obtained from a real-valued Gaussian distribution with zero mean and variance $d t$, and it is coupled to the diffusion tensor through the fluctuation-dissipation theorem, $\mathbf{D}=\mathbf{B} \cdot \mathbf{B}^{T}$; and finally, the second diffusive term, $\sqrt{2} \mathbf{B} \cdot d \mathbf{W}$, represents the Brownian displacement that results from collisions between mesh points and the surrounding (implicit) solvent.

The main challenge in simulating a stochastic process using Eq. (3) is the fact that the mobility tensor, $\mathbf{M}$, cannot be constructed explicitly under confinement for arbitrary geometries. This implies that the fluctuating velocity, $\mathbf{U}$, the divergence of the diffusion tensor, $\nabla \cdot \mathbf{D}$, and the diffusion tensor decomposition, $\mathbf{B}$, must be implemented in a way such that the scheme is matrix-free. To address this issue, we have developed an efficient $O(N)$ numerical algorithm, parallel Finite Element-General Geometry Ewald-like Method (pFEGgEm). ${ }^{28}$ The algorithm uses (i) the General geometry Ewald-like method $(\mathrm{GgEm})^{56}$ for a matrix-free product of the mobility tensor with any vector, $\mathbf{M} \cdot \mathbf{F}$; (ii) a mid-point algorithm, proposed by Fixman, ${ }^{57}$ that avoids the explicit calculation of $\nabla \cdot \mathbf{D}$; and (iii) a Chebyshev polynomial approximation for the $\mathbf{B} \cdot d \mathbf{W}$ product that uses $\mathrm{GgEm}$ to avoid the explicit calculation of $\mathbf{D}$. The algorithm is able to handle arbitrarily shaped confining walls.

Each particle in the simulation is represented by a discretized surface, and the force distribution on these moving particles is discretized into distribution of regularized point-forces. Using the Immersed Boundary (IB) method, ${ }^{58}$ the force distributions at these particles is discretized into a distribution of regularized point-forces. In particular,

$$
\boldsymbol{\rho}_{\mathrm{IB}}^{f}(\mathbf{x})=\sum_{v=1}^{N_{\mathrm{IB}}} \mathbf{f}_{v}^{\mathrm{C}} \delta_{\mathrm{IB}}\left(\mathbf{x}-\mathbf{x}_{v}\right),
$$

where $\mathbf{f}_{v}^{\mathrm{C}}$ represents the constitutive force acting on $v$ th surface node (point force with an excluded volume of radius $a$ ), $N_{\text {IB }}$ represents the number of surface nodes that are used to represent the suspended finite-size particles, $\delta_{\mathrm{IB}}$ is the modified Gaussian regularization function. The regularization parameter $\xi_{\mathrm{IB}}$ in $\delta_{\mathrm{IB}}$ is related to the characteristic length $h$ for the node spacing on the particle surface, i.e., $\xi_{\mathrm{IB}}$ $\sim h^{-1} \sim a^{-1}$. By doing this, we ensure that the regularized force on each node is spread over the length scale of the associated surface elements to prevent fluid from "penetrating" the particles. Further details about simulations are available in Refs. 28 and 30.

Here, we consider particles to be "semi-rigid," and the nodes on a particle are linked to each other by a spring, whose stiffness sets the rigidity of the particles. To conserve the shape of the particle, surface nodes are also connected to the center-of-mass of the particle by a spring. We assume each of these springs is linear, and the force acting on the point $v$ by the point $\mu$ is given by

$$
\mathbf{f}_{v \mu}^{\mathrm{C}}=k\left(r_{v \mu}-q_{0}\right) \frac{\mathbf{x}_{v \mu}}{r_{v \mu}},
$$

where $k$ and $q_{0}$ are the spring constant and the equilibrium spring length, respectively, for each specific node pair, $\mathbf{x}_{\nu \mu}=\mathbf{x}_{v}-\mathbf{x}_{\mu}$ and $r_{v \mu}$ $=\left|\mathbf{x}_{v \mu}\right|$.

The volume of spheres and that of cylinders are the same. Each surface node is linked to the neighboring node as well as to the center-of-mass point of the particle using an elastic spring with stiffness $k$. A spring network is formed for every particle, which results in an internal nodal force that resists deformation and maintains its shape. At the same time, a repulsive Lennard-Jones (LJ) potential is used for particle-particle and particle-wall excluded volume interactions. The ratio between mesh and particle size controls the number of surface nodes on each particle. Increasing the number of nodes improves accuracy but also increases the computational cost. 
In a previous study, ${ }^{30}$ we showed that a spring stiffness $k=200$ is sufficient to simulate "semi-rigid" particles, where despite the high concentration of particles, excluded volume interactions do not alter the particle shape. In addition, we found that particle discretization at the level of $N_{I B}=20$ is enough to avoid fluid penetration, satisfy Stokes' law, and provide the correct diffusional behavior. In this work, however, we use spheres and cylinders discretized with $N_{I B}=88$ to ensure extremely high accuracy.

In what follows, the characteristic units are $a$ for length, $a^{2} \zeta / k_{B} T$ for time, $k_{B} T$ for the energy, and $k_{B} T / a$ for the force. $\zeta$ the node friction coefficient is related to the fluid viscosity $\eta$ and $a$ through Stokes' law, i.e., $\zeta=6 \pi \eta a$, and the unit diffusivity, $D_{0}$, is defined as the diffusivity of a sphere in an infinite fluid with viscosity $\eta$, i.e., $D_{0}=k_{B} T / 6 \pi \eta a$.

\section{RESULTS}

We consider different particle mixtures of spheres and cylinders suspended in a Newtonian viscous fluid within a spherical cavity of radius $R=15$. The spherical particle has a radius $r_{s}=3$, leading to a hydrodynamic volume of $V_{\mathrm{HI}}=4 / 3 \pi r_{s}^{3}$. The cylinders have an aspect ratio of 2, i.e., $h_{c}=2 r_{c}$, where $r_{c}=2.62$ is the radius and $h_{c}$ is the height.

In our semi-rigid particle model, there are two ways to define the particle concentration in a cavity of volume $V$. A hydrodynamic volume fraction can be defined as $\phi_{\mathrm{HI}}=N_{T} V_{\mathrm{HI}} / V$; a second one is based on the excluded volume, $\phi_{\mathrm{EV}}=N_{T} V_{\mathrm{EV}} / V$, where for spheres and cylinders, we have $V_{\mathrm{EV}}=4 / 3 \pi\left(r_{S}+a\right)^{3}$ and $V_{\mathrm{EV}}$ $=\pi\left(r_{C}+a\right)^{2}\left(h_{C}+2 a\right)$, respectively. In the remainder of the article, we will use the hydrodynamic volume fraction $\phi_{\mathrm{HI}}$ (referred to as $\phi$ in the rest of the paper) to denote the concentration of the particles. In this work, we explore $\phi=[5 \%, 10 \%, 15 \%, 20 \%]$; this would correspond to $\phi_{\mathrm{EV}}=[12 \%, 24 \%, 36 \%, 48 \%]$. Figure 1 shows several details of our simulations and representative snapshots for $\phi=0.2$ with different cylinder fractions.

\section{A. Structure of sphere and cylinder mixture}

We begin by analyzing the structure of mixtures through the local particle number density. To calculate it, the spherical cavity is discretized into $m$ evenly spaced spherical shells along the radial direction, leading to a shell radius of the $i$ th shell that is given by $b_{i}$ $=(i+0.5) R / m$. The particle number density is then given by $n\left(r_{i}\right)$ $=\left\langle N\left(r_{i}\right) / V_{i}\right\rangle$, where $N\left(r_{i}\right)$ is the number of particles in the $i$ th shell with volume $V_{i}$ and is at a distance $r_{i}$ from the center of the cavity; \langle\rangle represents the ensemble average over time. We calculate the number density for all particles, only spheres, and only cylinders, and denote them by $n_{T}, n_{S}$, and $n_{C}$, respectively.

Figure 2 displays the number density for particles within the cavity for various particle concentrations $\phi$ and different fractions of cylinders for each $\phi$. Figure 2(a) shows the bare number density $n_{T}(r)$ for all particles within the cavity for various particle concentrations $\phi$ and different fractions of cylinders $\psi$. Cases with $\psi=0 \%$ and $100 \%$ refer to packings with pure spheres and cylinders, respectively. A common observation is that a peak in $n_{T}(r)$ appears close to the wall along with another peak close to the center of the cavity. These undulations in density $n_{T}$ are nearly absent for the case of $\phi=5 \%$ and grow with an increase in the volume fraction. For $\phi=10 \%, 15 \%$, the peak near the center grows taller with $\psi$. At the highest concentration of $\phi=20 \%$ considered here, we observe a layered structure with two distinct peaks at $r=3$ and 10 , along with a depletion zone in the regions $r<2$ and $5<r<7$. This depleted region is highlighted by the gray shaded region. Note that the position of the peak close to the center is nearly similar for the pure sphere and cylinder cases, and it shifts inwards for intermediate $\psi$ cases, hinting that the mixed cases exhibit a different structure from the pure cases. These observations are consistent with previous studies. ${ }^{27,30}$ It is important to mention that the layered structure has a strong dependence on the cavity size and more precisely on the relative size of the cavity and that of the particles, as shown previously. ${ }^{27,30}$

For a better comparison between all particle concentrations, in Fig. 2(b), we show the scaled number density $\mathrm{n}_{\mathrm{T}} / \max \left(\mathrm{n}_{\mathrm{T}}\right)$ for various particle concentrations $\phi$ and different fractions of cylinders $\psi$. Once again, cases with $\psi=0 \%$ and $100 \%$ are also shown. For a low concentration, $\phi=5 \%$, the peak in scaled number density near the wall as well as at the center is independent of $\psi$, while it decreases with $\psi$ in the bulk. For a particle concentration $\phi=10 \%$, the scaled density is higher at the center compared to the wall, which gets narrower with an increase in $\psi$. A similar observation can be drawn for the case with $\phi=15 \%$. Interestingly, for these two particle concentrations (especially for $\phi=15 \%$ ), the density of particles near the wall and the center of the cavity is most symmetric for the case of $\psi=0 \%$, i.e., the one with pure spheres. The difference between the two peaks actually increases with an increase in $\psi$, hinting that the alignment of cylinders can affect the way in which particles pack. This observation also implies that the addition of cylinders enhances the heterogeneity in the local packing or density. At the highest particle concentration $\phi=20 \%$, for the layered structure, the differences between the heights of the two peaks (at the center and wall of the cavity) actually decrease with $\psi$.

To further understand the local particle density, we analyze the relative density of spheres and cylinders. Figure 2(c) displays the number density of spheres relative to the total density as a function of $r$ for various values of $\phi$ and $\psi=25 \%, 50 \%$, and 75\% for each case. We observe that the scaled sphere density relative to the total number density $n_{S} / n_{T}$ is highest close to the wall and decreases with an increase in the fraction of cylinders. $n_{S} / n_{T}$ decreases with an increase in the fraction of cylinders in the bulk and is always greater than zero for low particle concentrations $\phi=5 \%$ and $10 \%$. $n_{S} / n_{T}$ becomes zero for higher particle concentrations $\phi=15 \%$ and $20 \%$ at large cylinder fraction $(75 \%)$; only cylinders are found in this range of $r$, as confirmed by $n_{C} / n_{T}$ being equal to 1 , as shown in Fig. 2(d). These two observations demonstrate that only cylinders are present in the interior of the cavity and that spheres are close to the wall. Another point to note is that the numerical values of the scaled densities for $\psi=25 \%$ and $50 \%$ in the bulk are more "separated" compared to the differences between $\psi=50 \%$ and $75 \%$.

These results serve to establish the equilibrium segregation of spheres to the walls induced by a subtle difference in particle shape but for the same particle volume. As a side note, we mention here that the difference in particle volume may not be the only reason for the observed segregation in experiments.

Next, we analyze the orientational order parameter for different particle concentrations and cylinder fractions. The orientational 

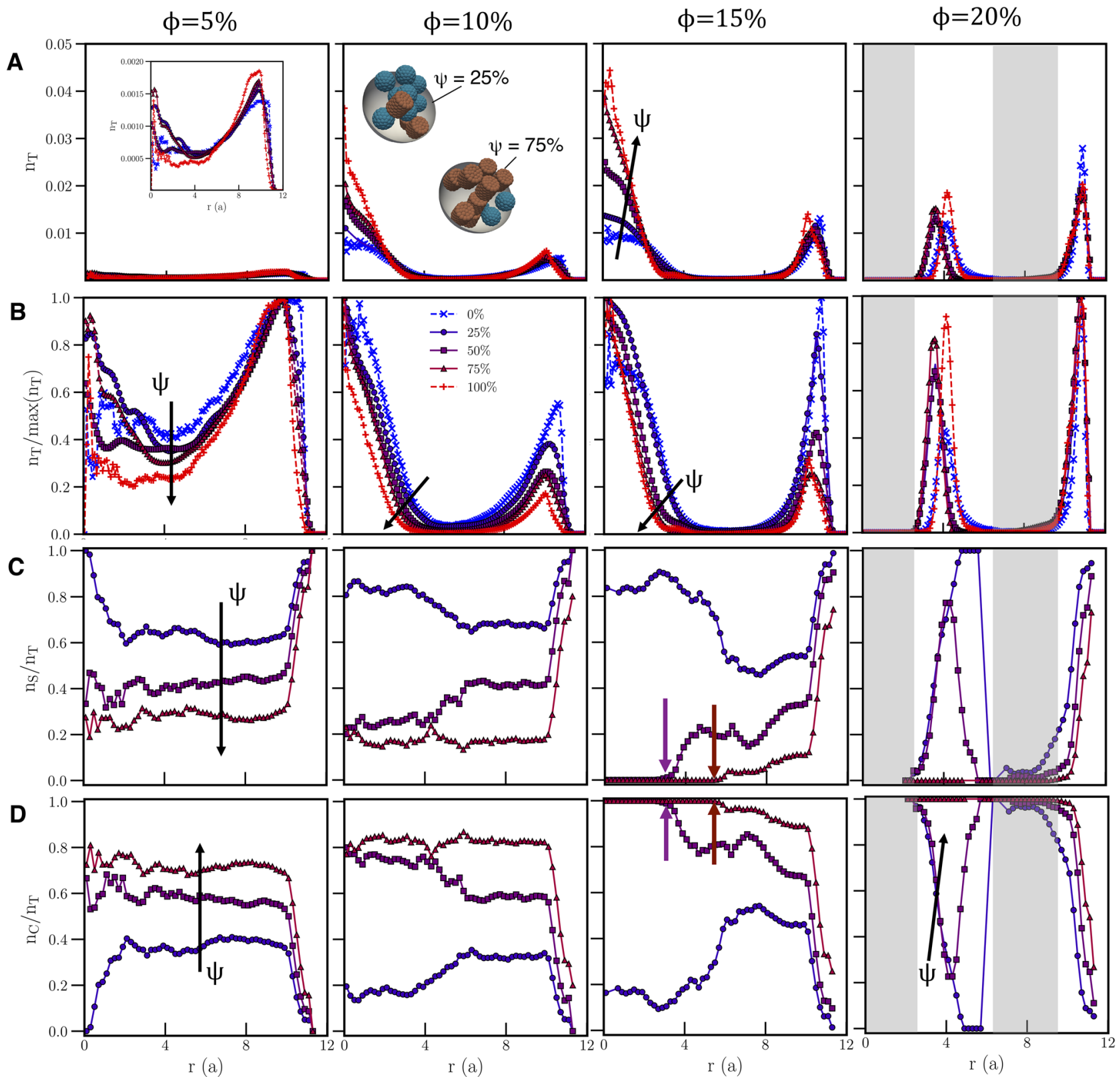

FIG. 2. Particle number density in the mixture of spherical and cylindrical particles within a spherical cavity of radius $R=15$ as a function of radial distance. The radii of spheres and cylinders are $r_{S}=3$ and $r_{C}=2.62$, respectively. The cylinder has an aspect ratio of 2, i.e., $h_{C}=2 r_{C}=5.24$. (a) Number density of all particles scaled with the maximum density $n_{T} / \max \left(n_{T}\right)$ for particle concentrations $\phi=5 \%, 10 \%, 15 \%$, and $20 \%$ (from left to right). (b) Relative number density of all particles scaled with the maximum density $n_{T} / \max \left(n_{T}\right)$ for particle concentrations $\phi=5 \%, 10 \%, 15 \%$, and $20 \%$ (from left to right). For each particle concentration, different fractions of cylinders $\psi$ are displayed along with the pure sphere $(0 \%)$ and pure cylinder $(100 \%)$ cases. (c) Relative number density of only spherical particles scaled with the total density $n_{S} / n_{T}$ for various particle concentrations $\phi=5 \%, 10 \%, 15 \%$, and $20 \%$ (from left to right). (d) Relative number density of only cylindrical particles scaled with the total density $\mathrm{n}_{\mathrm{C}} / \mathrm{n}_{\mathrm{T}}$ for various particle concentrations $\phi=5 \%, 10 \%, 15 \%$, and $20 \%$ (from left to right).

order parameter is defined as $\lambda=\frac{1}{2}\left\langle 3 \cos ^{2} \theta-1\right\rangle$, where $\cos \theta=\mathbf{m} \cdot \mathbf{n}$ $/(\|\mathbf{m}\| \cdot\|\mathbf{n}\|), \mathbf{m}$ is the vector parallel to the centerline of the cylinder and $\mathbf{n}$ is the vector connecting the cavity center and the cylinder's center-of-mass. A parameter $\lambda$ is often used in liquid crystalline systems to quantify the nematic ordering; ${ }^{59,60} \lambda=0$ corresponds to a random/disordered configuration, whereas $\lambda$ is unity for ordered morphologies, with the cylinder axis being coaxial with the radial direction of the spherical cavity (radial phase), and $\lambda=-1 / 2$ when 
all cylinders are aligned transversal to the radial direction (concentric phase). Figure 3 displays $\lambda$ for various particle concentrations with different fractions of cylinders. A common observation is that, very close to the wall, the order parameter is $\lambda=-1 / 2$ irrespective of the volume fraction, indicating a concentric phase close to the wall. We also find that $\lambda$ fluctuates around zero in the bulk for low volume fractions, i.e., $\phi=5 \%, 10 \%$, indicating a disordered configuration of cylinders. For moderate concentrations, $\phi=15 \%, \lambda$ is zero close to center and is negative with an increase in $r$, reaching a minimum and increasing further with $r$ to reach a maximum value of $0.5 ; \lambda$ then decreases with $r$ reaching -0.5 close to the wall. For the highest concentration, we find that $\lambda=-1 / 2$ both at the center and close to the wall, and we also find a depletion zone with no particles for $r<2$. For this concentration, we find another ordered state with $\lambda \sim 1$ in the region $7<r<10$. The ordered morphology arises from segregation in the cavity. At the highest volume fraction $\phi=20 \%$, the cylinders display ordered morphologies, i.e., perpendicular to the radial direction very close to the wall and parallel to the radial direction for $7<r<10$. With an increase in $\psi$, the cylinders push the spheres to the wall in order to minimize free volume and gain orientational order.

Excluded volume potential calculations yield $10.88 k_{B} T$ for a single sphere, $2.3 k_{B} T$ for a cylinder oriented perpendicular to the cavity wall, and $1.01 k_{B} T$ for a cylinder oriented parallel to the cavity wall. These numbers imply that the cylinder oriented parallel to the cavity wall $(\lambda=-0.5)$ would be the most preferable configuration, which explains $\lambda=-0.5$ irrespective of the volume fraction $\phi$ and fraction of cylinders $\psi$.
We have shown that the confinement induces a structure that propagates from the cavity wall toward the center, whose length scale is not only controlled by the particle concentration but also by their shape. At low volume fractions, both the spheres and the cylinders are found in the bulk with cylinders oriented parallel to the cavity wall and a disordered configuration in the bulk. As the volume fraction increases, the cylinders at the wall still prefer to be oriented parallel to the cavity wall. An ordered configuration begins to emerge adjacent to this layer, that aligns almost perpendicular to the layer at the wall. Next, in an ordered state that is concentrically aligned along the radial direction (similar to that at the wall), these cooperative effects in the ordering of cylinders in the bulk cause the spheres to segregate to the cavity wall. Also note that, even though we demonstrate the layering of particles in the density profiles and structure in the orientational order parameter, the system is not crystalline; instead, it is still fluid-like, and particles diffuse throughout the system, as discussed in Sec. III B.

\section{B. Local mobility of the particles in the cavity}

As mentioned earlier, recent experiments ${ }^{61-65}$ and simulations $^{26,27}$ suggest that confinement can lead to anisotropic selfdiffusion, which is not the case for unconfined suspensions. In an unconfined suspension, the mobility depends only on the volume fraction and the absolute position of particles does not contribute and all relative separations between particles are equally probable. To examine this, mobilities (short-time diffusivity) in both the radial and tangential directions are calculated using the Einstein-Stokes
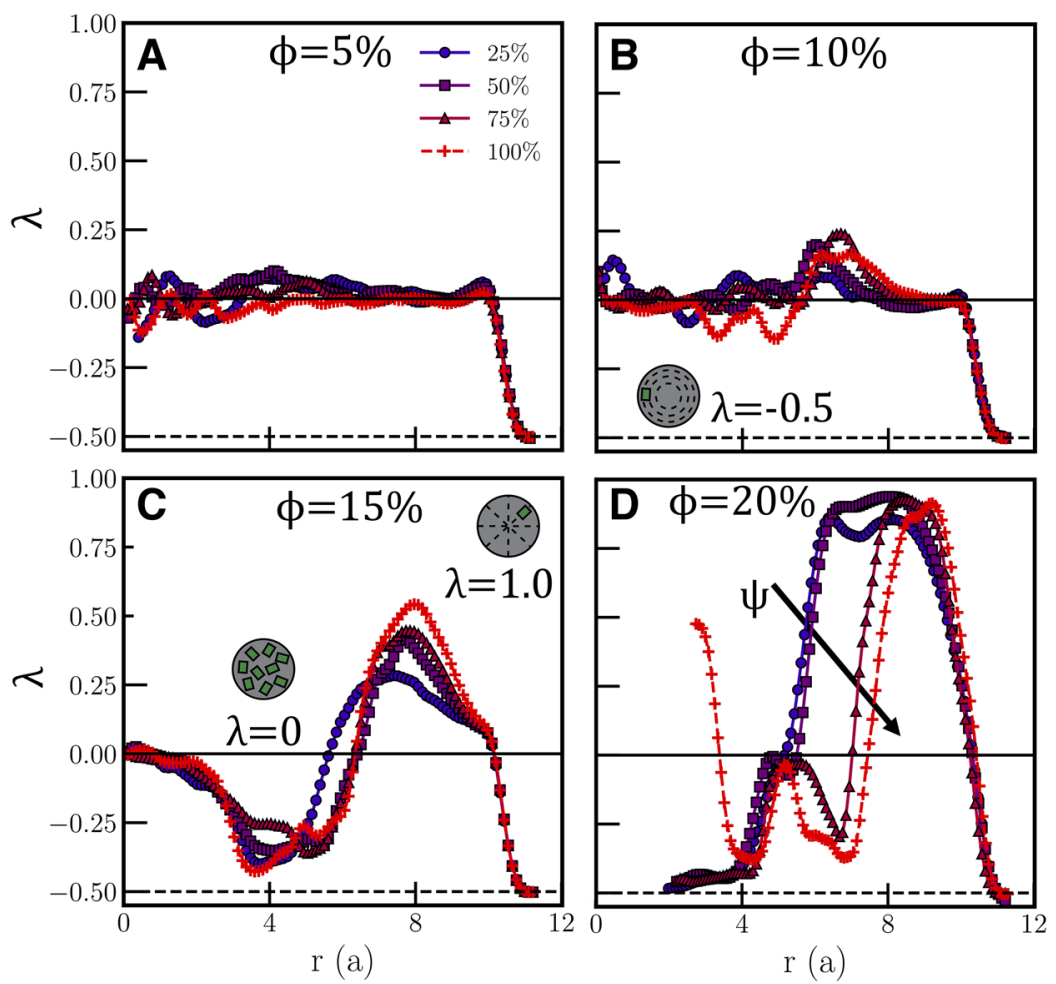

FIG. 3. Orientational order parameter $\lambda$ of cylindrical particles within a spherical cavity of $R=15$ as a function of radial distance for particle concentration $\phi=$ (a) $5 \%$, (b) $10 \%$, (c) $15 \%$, and (d) $20 \%$. The radius of cylinders are $r_{C}=2.62$ and the height $h_{C}=2 r_{C}=5.24$. 
relation, ${ }^{63}$

$$
\begin{aligned}
& \left\langle\Delta \mathbf{x}_{\mathrm{R}}^{2}(t)\right\rangle\left(r_{i}\right)=2 M_{\mathrm{R}}\left(r_{i}\right) d t, \\
& \left\langle\Delta \mathbf{x}_{\mathrm{T}}^{2}(t)\right\rangle\left(r_{i}\right)=4 M_{\mathrm{T}}\left(r_{i}\right) d t,
\end{aligned}
$$

for short time $t \rightarrow 0 ; \Delta \mathbf{x}=\mathbf{x}(t+d t)-\mathbf{x}(\mathrm{t}), \Delta \mathbf{x}_{\mathrm{R}}=\Delta \mathbf{x} \cdot \mathbf{x} /|\mathbf{x}|, \Delta \mathbf{x}_{\mathrm{T}}$ $=\Delta \mathbf{x}-\Delta \mathbf{x}_{\mathrm{R}}$ denote the radial and tangential displacements, respectively. $M_{\mathrm{R}}\left(r_{i}\right)$ and $M_{\mathrm{T}}\left(r_{i}\right)$ correspond to the instantaneous radial and tangential mobilities at radial location $r_{i}$ in an infinitesimal time interval $d t$. Instantaneous radial and tangential mobilities are averaged in each shell during a simulation and then over ten independent realizations. It is important to note that when the segregation is observed, the segregated region still consists of particles (either spheres or cylinders).

Figure 4 displays both the radial $M_{\mathrm{R}}$ and tangential $M_{\mathrm{T}}$ components of mobility within the cavity for mixtures of spherical and cylindrical particles as a function of radial distance for various particle concentrations. Note that the two components are normalized by the mobility of a spherical particle at infinite dilution $M_{00}$. A few observations can be drawn: mobilities along both directions are not constant along the radial direction; instead, the particles diffuse fastest at the cavity center and slowest at the cavity wall due to lubrication forces between wall and particles. Second, both $M_{\mathrm{R}}$ and $M_{\mathrm{T}}$ decrease with the increase in particle concentration due to enhanced many-body hydrodynamic interactions with $\phi$. Next, the peaks and trough in mobility appear at the same radial position, corresponding to the local particle density as shown in Fig. 2, thereby revealing a correlation between structure and dynamics. The strength of these undulations enhance with volume fraction that becomes particularly apparent for the case of $\phi=20 \%$, where a layered structure for both $M_{\mathrm{R}}$ and $M_{\mathrm{T}}$ corresponds to a similar density profile as observed in Fig. 2, e.g., the dip in mobility at $2<r<7$ corresponds to the peak in $\mathrm{n}_{\mathrm{T}}$ in the same radial range. Also note that for the case of $\phi=20 \%$, especially at $\psi=75 \%, 100 \%$, there is a discontinuity in the data due to the depletion zone observed in the particle density profile. Both $M_{\mathrm{R}}$ and $M_{\mathrm{T}}$ display the expected decrease with an increase in $\phi$ close to the wall for all cylinder fractions. For $\phi=5 \%$ and $10 \%$, we observe an expected decrease in mobility with an increase in $\phi$ in the bulk as well. In contrast, for higher particle concentrations $(\phi=15 \%)$, the mobility does not exhibit a decrease with $\phi$ in the bulk, and the mobility for $\phi=20 \%$ at radial location $r \sim 5$ becomes equal or even larger than that for $\phi=15 \%$. Also note that the undulations (or wavy behavior) observed in the radial mobility are stronger compared to those in the tangential component, signifying that the structure (density) has a stronger impact on the radial mobility. Physically, in a locally dense environment, the presence of neighboring particles will strongly hinder the radial motion as compared to the tangential motion. This explains why the presence of a particle (or absence of it in a depleted zone) causes larger variations in radial than tangential mobility.

The effects of changing cylinder fraction $\psi$ for a constant volume fraction $\phi$ are considered next. In Fig. 7, the data plotted in Fig. 4 are replotted to emphasize the effect of $\psi$ on both components of mobility for a constant $\phi$. Figure 7 illustrates that an increase in $\psi$ systematically decreases the mobility $M_{R}$ even for the smallest particle concentration $\phi=5 \%$. This trend is clearly observed for all volume fractions close to the wall. However, in the bulk, the observed trend is less clear for the highly concentrated cases $(\phi=15 \%, 20 \%)$. Especially for $\phi=20 \%$, the mobility
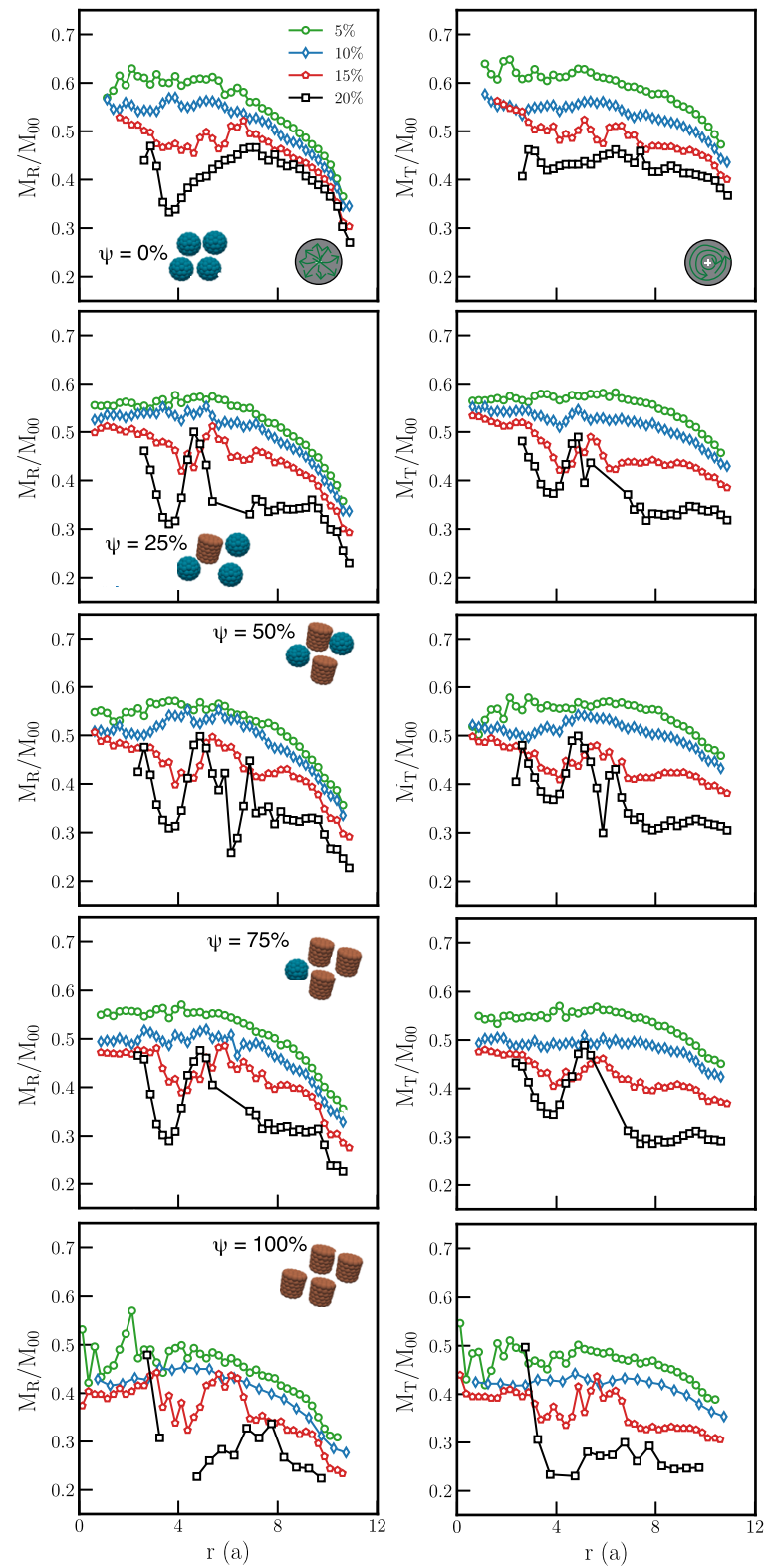

FIG. 4. Radial mobility $M_{R}$ (left) and tangential mobility $M_{T}$ (right) for mixture of cylindrical particles with $r_{C}=2.62$ and $h_{C}=2 r_{C}=5.24$ and spherical with $r_{S}=3$ confined in a spherical cavity of $R=15$ for various particle concentrations, $\phi$, and different fraction of cylinders $\psi$. Both components of mobility are normalized by the mobility of spherical particles in the bulk at infinite dilution $M_{00}$ for $t \rightarrow 0$. Note that for both mobilities, the data for $r / a<2.3$ are missing for $\phi=20 \%$, and the reasoning for this is that due to the layering structure, there are not enough particles to sample. The results with statistical error (error bars) are presented in Fig. 6.

in the region close to the center is insensitive to $\psi$, and in a small region, the mixture mobility is larger than that of pure spheres. Taken together, these observations reveal that the change in shape, i.e., the breaking of geometrical symmetry, affects the short-time 
diffusion. Recall that the cylindrical and spherical particles have equal volume, and the mobilities are normalized by the bulk mobility of a spherical particle. In summary, the short-time mobility in a confined suspension is position-dependent, anisotropic, and heterogeneous by heterogeneity in the structure induced by confinement. Here, we find that introducing cylinders enhances all these features.

\section{Long-time mobility of the particles}

The displacement of a Brownian particle in a confined system is hindered, and thus, the mean square displacement (MSD) over time is lower than that observed in a bulk system. ${ }^{25,27}$ Our recent work on pure suspensions ${ }^{30}$ showed that a change in shape from spherical to cylindrical at constant volume fraction leads to slower particle diffusion. The question that arises here is how does the fraction of cylinders in a mixture affect long-time dynamics.

Figure 5(a) shows the average MSDs for mixtures at different particle concentrations. The MSD of each system is calculated from ten independent simulations in which the particles diffuse for more than 300 particle diffusion times within the cavity. A few observations can be drawn. First, the MSDs grow linearly as short times, $t \rightarrow 0$, and reach a plateau in the long-time limit, $t \rightarrow \infty$. Second, at intermediate times, particle motion becomes sub-diffusive for systems with $\phi \geq 10 \%$.

To analyze the diffusion behavior in detail, we express the MSD as the generalized Stokes-Einstein relation:

$$
\left\langle(\mathbf{R}(t)-\mathbf{R}(0))^{2}\right\rangle=M_{i} t^{i},
$$

where $\mathbf{R}$ is the $3 N_{p}$ particle coordinate vector, $M_{i}$ is the generalized particle mobility coefficient, and $i$ is the power law exponent that characterizes whether the particle motion is sub-diffusive $(\alpha<1)$, diffusive $(\alpha=1)$, or super-diffusive $(\alpha>1)$. For the case of $(\alpha=1)$, the mobility $M_{0}$ is the diffusion coefficient.

Figures 5(b)-5(e) show our findings for the mean square displacement. Short-time mobility [Fig. 5(b)] and sub-diffusive mobility [Fig. 5(c)] decrease with an increase in the cylinder fraction $\psi$ and particle concentration $\phi$. The dependence of $M_{0} / M_{00}$ and $M_{\alpha} / M_{00}$ on particle concentration $\phi$ is monotonic; however, the dependence on cylinder fraction is weak at smaller cylinder fraction $\psi$ and becomes strong beyond 50\%, showing a smooth to "rigid" transition at $\psi=50 \%$. Note that for the case of $\phi=5 \%$, sub-diffusion is not observed at any cylinder fraction $\psi$. On the other hand, the sub-diffusive exponent $\alpha$ [Fig. 5(d)] that characterizes the strength of sub-diffusive behavior decreases with both $\phi$ and $\psi$. In the case of short-time and sub-diffusive mobilities $M_{0} / M_{00}$ and $M_{\alpha} / M_{00}$, the dependence on $\psi$ is monotonic; however, the correlation gets less pronounced with an increase in the particle concentration. Finally, the transition time, defined as the time at which the system transitions from the short-time diffusive to the intermediate time subdiffusive regime, is displayed in Fig. 5(e). We observe that for $\phi=0.1$, the transition time decreases strongly with the cylinder fraction and becomes nearly independent of cylinder fraction for higher particle concentrations.

From a physical perspective, the particle dynamics can be characterized by different time scales that correspond to different regimes of the motion of particle. ${ }^{66}$ At short times, molecular diffusion dominates, and hence, particle motion is diffusive. As the diffusing particle probes the surrounding fluid and other particles, it distorts the suspension structure that leads to correlated motion, and eventually hydrodynamic interactions (HI) begin to dominate. At finite concentrations, interparticle hydrodynamic interactions (HI) dominate leading to a slowdown of exchange of particle positions and their motion, leading to the sub-diffusive behavior at
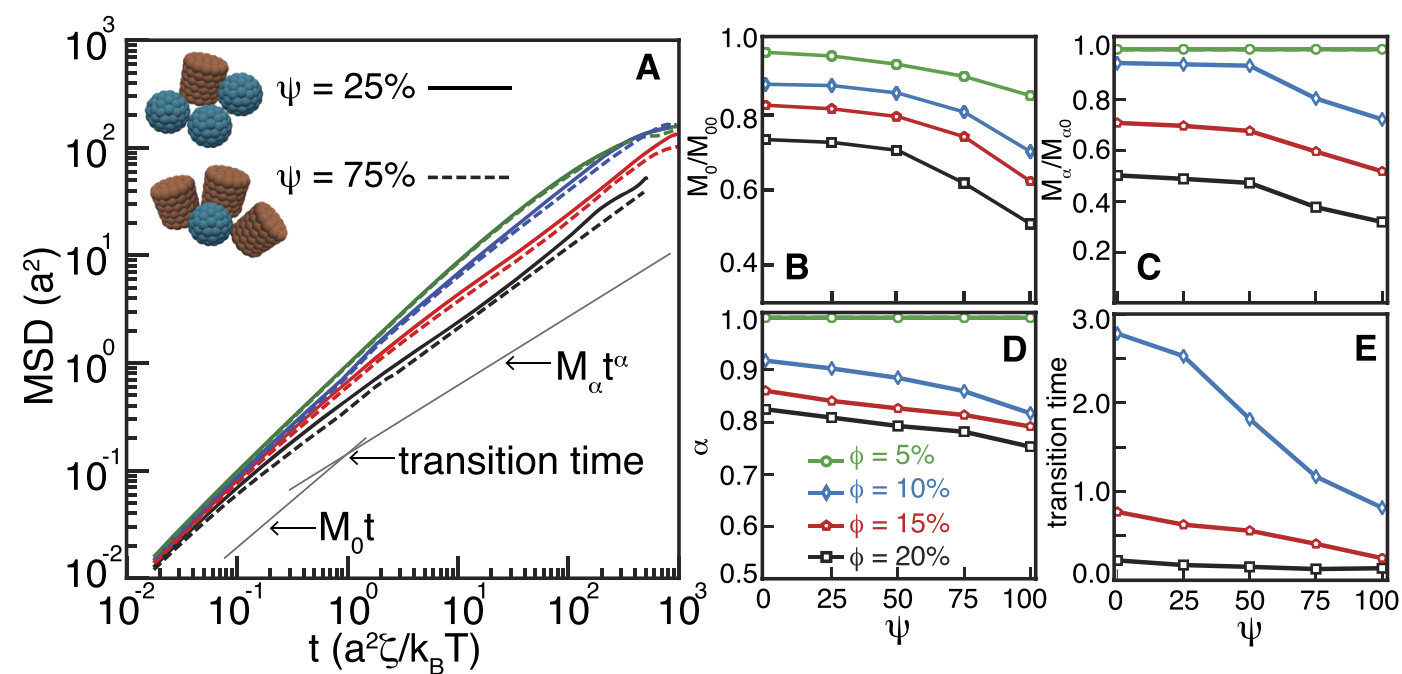

FIG. 5. (a) Mean square displacement as a function of time for mixture of spherical and cylindrical particles that are confined in a spherical cavity with $R=15$ for various particle concentrations $\phi$ with varying fraction of cylinders. Solid and dashed lines are the results for $\psi=25 \%$, and $75 \%$ fraction of cylinders; (b) short-time mobility scaled with the mobility of spherical particles in bulk at infinite dilution $M_{00}$ plotted against $\psi$, (c) sub-diffusive (at intermediate time) mobility scaled with the mobility of spherical particles in bulk at infinite dilution $M_{00}$ plotted against $\psi$, (d) sub-diffusive exponent $\alpha$ plotted against $\psi$, and (e) time scale to make transition between diffusive to sub-diffusive behavior plotted as a function of fraction of cylinders for various values of $\phi$ 
intermediate times. At $t \rightarrow \infty$, for all values of $\phi$ and $\psi$, the confinement due to the cavity wall leads to the long-time plateau. Previous work has shown that crowding exacerbated by short-range hydrodynamic interactions (lubrication) is a key contributor to the sub-diffusive behavior. ${ }^{30}$ With an increase in $\phi$, the crowding leads to slowing of diffusion at short times. At intermediate times, when particles begin to move relative to each other, the net effect of $\mathrm{HI}$ is enhanced with an increase in the volume fraction (due to multi body interactions), and hence, the extent of sub-diffusive behavior becomes more pronounced. Further, to explain the smooth to rigid transition as observed in both $M_{0} / M_{00}$ and $M_{\alpha} / M_{00}$, we refer to the scaled density profiles with an increase in $\psi$. We observe that the scaled densities $n_{T} / \max \left(n_{T}\right)$ for $\psi=0 \%$ and $25 \%$ are similar but then drop drastically for $\psi \geq 50 \%$, which affects the mobility in both the diffusive and sub-diffusive cases. For the case of $\phi=5 \%$, since the cylinders are in a disordered state in the bulk, i.e., $\lambda=0$, the dependence of these quantities on $\psi$ is minimal. Some structure begins to appear, as evidenced by orientational order parameter for high volume fractions $\phi=15 \%, 20 \%$, especially for $\psi=75 \%$, $100 \%$, leading to a drastic slowdown of the particles that manifests itself in a drastic slowdown in mobilities for $\psi \geq 75 \%$. On the other hand, during the transition time between the diffusive and the sub-diffusive regimes (i.e., roughly the time needed for particles to diffuse a distance nearly equal to their radius), collisions between particles slow down their motion, leading to sub-diffusive behavior. It follows that the transition time decreases with an increase in the particle concentration $\phi$. At low $\phi$, changing the shape from spheres $(\psi=0 \%)$ to $(\psi=100 \%)$ to cylinders, the larger aspect ratio of the latter should cause them to feel each other at shorter time scales, compared to what is seen for spheres and hence yield a transition time that decreases with $\psi$. At larger $\phi$, the system is so dense that even for different packings, i.e., sphere-sphere, sphere-cylinder, and cylinder-cylinder cases, the particle interaction time scales become similar.

\section{CONCLUSIONS}

We have examined the structure and dynamics of mixtures of finite size in mixtures of spherical and cylindrical particles confined in a spherical cavity. An Immersed Boundary-General geometry Ewald-like Method (IB-GgEm) approach was used in the corresponding calculations, thereby taking into account hydrodynamic interactions between particles and between particles and confining walls. By systematically varying the cylinder fraction at different particle concentrations, it was found that particle shape has a pronounced effect on both the structure and short- and longtime dynamics of confined Brownian suspensions. As the volume fraction increases, the effect of cylinder fraction becomes more evident. At short times, the diffusion of particles in the confined cavity is anisotropic and depends strongly on the distance from the cavity center. At long times, the mean-squared displacement reaches a plateau, and at intermediate times, a sub-diffusive behavior is observed, which shows a pronounced dependence on cylinder fraction $\psi$.

Our results suggest that introducing non-spherical particles affects the local structure and local dynamics and global dynamics in different ways. At a local level, particles are found to segregate based on shape, with cylinders adopting conformations with high orientational order. At a global level, cylinders give rise to pronounced differences in the short-time mobility, the sub-diffusive behavior at intermediate time scales, and the transition time from diffusive to sub-diffusive behavior. Particle concentration has a strong effect
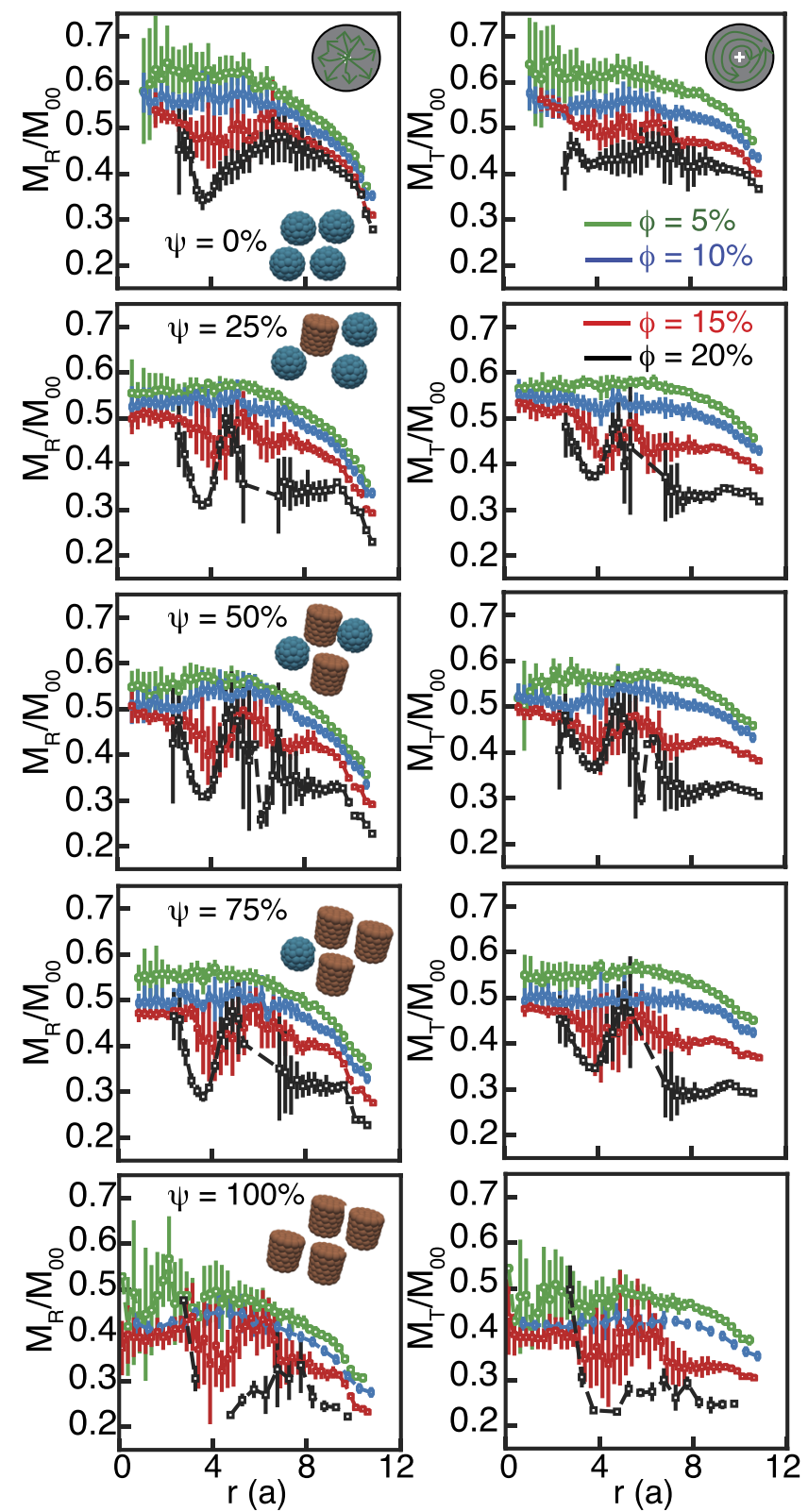

FIG. 6. Statistical errors in mobility calculations: radial mobility $M_{R}$ (left) and tangential mobility $M_{T}$ (right) for mixture of cylindrical particles with $r_{C}=2.62$ and $h_{C}=2 r_{C}=5.24$, and spherical with $r_{S}=3$ confined in a spherical cavity of $R$ $=15$ for various particle concentrations, $\phi$, and different fraction of cylinders $\psi$. Both components of mobility are normalized by the mobility of spherical particles in the bulk at infinite dilution $M_{00}$ for $t \rightarrow 0$. The error bars represent the statistical error. 
on such transitions. To start with, the mobility shows a smooth to stiff transition at $50 \%$ cylinder fraction for all particle concentrations and the sharpness of this transition increases with particle concentration. Second, the sub-diffusive slope and sub-diffusive exponent show mixed features as a function of cylinder fraction. The slope shows a smooth to stiff transition similar to that of the shorttime mobility. The exponent decreases smoothly with an increase in the cylinder fraction. In both cases, the dependence on the cylinder fraction is insensitive to particle concentration. Finally, the transition time from the diffusive to sub-diffusive regime depends strongly on the cylinder fraction for low particle concentrations $(\phi$ $=0.1$; that dependence weakens with an increase in the particle concentration.

In terms of practical applications of this work, we have demonstrated that a subtle change in shape, i.e., keeping the same volume but breaking the symmetry, can bring about profound differences in the structural and dynamic properties of a confined suspension. As mentioned in the Introduction, a basic understanding of diffusion, and in particular the anomalous diffusion, is highly relevant to several scientific and practical applications such as living cells, mesoporous materials, and energy generation processes. ${ }^{1-3,9,10}$ The approach developed here could shed further light on the diffusion of highly concentrated biomolecules. ${ }^{11-15}$ Our work should be of special relevance to the understanding of diffusion in the cytoplasm, where proteins exhibit a wide distribution of sizes and shapes. ${ }^{67}$ Changes in shape while keeping a constant volume fraction have not been considered before. Here, we show that this symmetry breaking leads to segregation in the cavity by inducing orientational ordering in the system. Our study also highlights the importance of shape on non-Newtonian features that are generally observed in colloidal suspensions, ${ }^{68-73}$ where shape shows strong effects. ${ }^{7}$

As an outlook, the role of aspect ratio and electrostatic interaction on particle mobility in confined mixtures will be considered in future that arise in a realistic cell environment.

\section{AUTHOR'S CONTRIBUTIONS}

A.S. and J.L. contributed equally to this work.

\section{ACKNOWLEDGMENTS}

We would like to thank Vivek Sharma, Grayson Jackson, and Miachel van der Naald for fruitful discussions. The development of fast computational codes for simulations of nanoparticles interacting through hydrodynamic and polarization interactions was supported by the Department of Energy, Basic Energy Sciences, Division of Materials Research, through the MICCoM center and Award No. DOE-SC0008631 (KFF). The calculations on particle segregation and transport presented in this work were supported by the Department of Energy, Basic Energy Sciences, Division of Materials Research, through the AMEWS Center. The authors gratefully acknowledge the computing resources provided
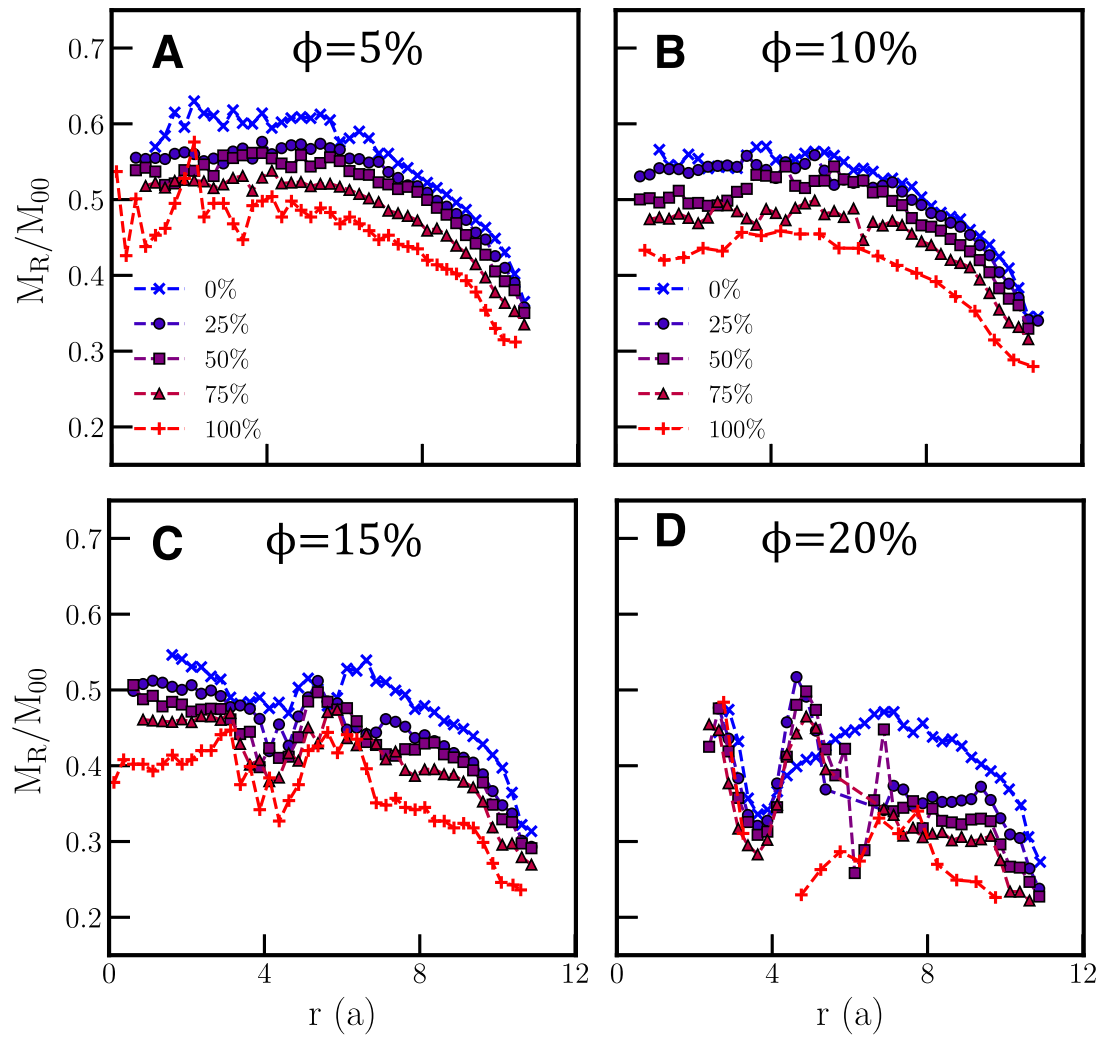

FIG. 7. Radial mobility $M_{R}$ for mixture of cylindrical particles with $r_{C}=2.62$ and $h_{C}=2 r_{C}=5.24$ and spherical particles with $r_{S}=3$ confined in a spherical cavity of $R=15$ for particle concentration $\phi=$ (a) $5 \%$, (b) $10 \%$, (c) $15 \%$, and (d) $20 \%$. The radial mobility is normalized by the mobility of spherical particles in the bulk at infinite dilution $M_{00}$ for $t \rightarrow 0$. 
on Bebop (Blues), a high-performance computing cluster operated by the Laboratory Computing Resource Center at Argonne National Laboratory, and the University of Chicago Research Computing Center.

\section{APPENDIX: LOCAL MOBILITY OF THE PARTICLES}

Figure 6 displays both the radial $M_{\mathrm{R}}$ and the tangential $M_{\mathrm{T}}$ components of mobility within the cavity for mixtures of spherical and cylindrical particles as functions of radial distance with error bars (statistical error) for various particle concentrations. The error bars in the data are small at the wall but systematically increase as the radial distance decreases toward the center for even the smallest particle concentration $\phi=5 \%$ simulated here. This is primarily due to fluctuations in the structure (density) in the bulk of the cavity. Especially interesting is the case of the highest particle concentration $\phi=20 \%$, where error bars are the largest in the depleted region. The observed trend, in which the error bars are larger close to the center, is consistent with the literature ${ }^{27,30}$ and is due to fewer number of particle available in the region leading to sampling error reflected here.

\section{REFERENCES}

${ }^{1} \mathrm{~J}$. Happel and H. Brenner, Low Reynolds Number Hydrodynamics: With Special Applications to Particulate Media (Prentice-Hall, Englewood Cliffs, New Jersey, 1965).

${ }^{2}$ D. L. Koch and G. Subramanian, "Collective hydrodynamics of swimming microorganisms: Living fluids,” Annu. Rev. Fluid Mech. 43, 637-659 (2011).

${ }^{3}$ L. K. Hudson, J. Eastoe, and P. J. Dowding, "Nanotechnology in action: Overbased nanodetergents as lubricant oil additives," Adv. Colloid Interface Sci. 123-126, 425-431 (2006).

${ }^{4} \mathrm{R}$. Richert, "Geometrical confinement and cooperativity in supercooled liquids studied by solvation dynamics," Phys. Rev. B 54, 15762 (1996).

${ }^{5}$ J. D. Seymour, J. P. Gage, S. L. Codd, and R. Gerlach, "Anomalous fluid transport in porous media induced by biofilm growth,” Phys. Rev. Lett. 93, 198103 (2004).

${ }^{6} \mathrm{~J}$. K. Dhont, An Introduction to Dynamics of Colloids (Elsevier, 1996).

${ }^{7}$ J. Gao, W. Guo, D. Feng, H. Wang, D. Zhao, and L. Jiang, "High-performance ionic diode membrane for salinity gradient power generation," J. Am. Chem. Soc. 136, 12265-12272 (2014).

${ }^{8}$ Z. Zhang, X. Sui, P. Li, G. Xie, X.-Y. Kong, K. Xiao, L. Gao, L. Wen, and L. Jiang, "Ultrathin and ion-selective janus membranes for high-performance osmotic energy conversion," J. Am. Chem. Soc. 139, 8905-8914 (2017).

${ }^{9}$ S. B. Darling, "Perspective: Interfacial materials at the interface of energy and water," J. Appl. Phys. 124, 030901 (2018).

${ }^{10}$ R. Z. Waldman, H.-C. Yang, D. J. Mandia, P. F. Nealey, J. W. Elam, and S. B. Darling, "Janus membranes via diffusion-controlled atomic layer deposition," Adv. Mater. Interfaces 5, 1800658 (2018).

${ }^{11}$ A. P. Minton, "Excluded volume as a determinant of macromolecular structure and reactivity," Biopolymers 20, 2093 (1981).

${ }^{12}$ A. B. Fulton, "How crowded is the cytoplasm?," Cell 33, 345-347 (1982).

${ }^{13}$ M. C. Konopka, I. A. Shkel, S. Cayley, M. T. Record, and J. C. Weisshaar, "Crowding and confinement effects on protein diffusion in vivo," J. Bacteriol. 188, 6115-6123 (2006).

${ }^{14}$ R. J. Ellis, "Macromolecular crowding: Obvious but underappreciated," Trends Biochem. Sci. 26, 597-604 (2001).

${ }^{15}$ R. J. Ellis, "Macromolecular crowding: An important but neglected aspect of the intracellular environment," Curr. Opin. Struct. Biol. 11, 114-119 (2001).

${ }^{16}$ M. Grimaldo, H. Lopez, C. Beck, F. Roosen-Runge, M. Moulin, J. M. Devos, V. Laux, M. Härtlein, S. Da Vela, R. Schweins, A. Mariani, F. Zhang, J.-L. Barrat, M. Oettel, V. T. Forsyth, T. Seydel, and F. Schreiber, "Protein short-time diffusion in a naturally crowded environment," J. Phys. Chem. Lett. 10, 1709-1715 (2019).
${ }^{17}$ C. Selhuber-Unkel, P. Yde, K. Berg-Sørensen, and L. B. Oddershede, "Variety in intracellular diffusion during the cell cycle," Phys. Biol. 6, 025015 (2009).

${ }^{18}$ M. Weiss, M. Elsner, F. Kartberg, and T. Nilsson, "Anomalous subdiffusion is a measure for cytoplasmic crowding in living cells," Biophys. J. 87, 3518-3524 (2004).

${ }^{19} \mathrm{~F}$. Höfling and T. Franosch, "Anomalous transport in the crowded world of biological cells,” Rep. Prog. Phys. 76, 046602 (2013).

${ }^{20}$ D. J. Bicout and M. J. Field, "Stochastic dynamics simulations of macromolecular diffusion in a model of the cytoplasm of Escherichia coli," J. Phys. Chem. 100, 2489-2497 (1996).

${ }^{21}$ S. R. McGuffee and A. H. Elcock, "Diffusion, crowding \& protein stability in a dynamic molecular model of the bacterial cytoplasm," PLoS Comput. Biol. 6, e1000694 (2010).

${ }^{22}$ A. Daddi-Moussa-Ider, A. Guckenberger, and S. Gekle, "Particle mobility between two planar elastic membranes: Brownian motion and membrane deformation," Phys. Fluids 28, 071903 (2016).

${ }^{23}$ A. S. Sangani, A. Acrivos, and P. Peyla, "Roles of particle-wall and particleparticle interactions in highly confined suspensions of spherical particles being sheared at low Reynolds numbers," Phys. Fluids 23, 083302 (2011).

${ }^{24} \mathrm{~T}$. Ando and J. Skolnick, "Crowding and hydrodynamic interactions likely dominate in vivo macromolecular motion," Proc. Natl. Acad. Sci. U. S. A. 107, 18457-18462 (2010).

${ }^{25}$ E. Chow and J. Skolnick, "Effects of confinement on models of intracellular macromolecular dynamics," Proc. Natl. Acad. Sci. U. S. A. 112, 14846-14851 (2015).

${ }^{26}$ C. Aponte-Rivera and R. N. Zia, "Simulation of hydrodynamically interacting particles confined by a spherical cavity," Phys. Rev. Fluids 1, 023301 (2016).

${ }^{27}$ C. Aponte-Rivera, Y. Su, and R. N. Zia, "Equilibrium structure and diffusion in concentrated hydrodynamically interacting suspensions confined by a spherical cavity," J. Fluid Mech. 836, 413 (2018).

${ }^{28}$ X. Zhao, J. Li, X. Jiang, D. Karpeev, O. Heinonen, B. Smith, J. P. HernandezOrtiz, and J. J. de Pablo, "Parallel $O(N)$ Stokes' solver towards scalable Brownian dynamics of hydrodynamically interacting objects in general geometries," $\mathrm{J}$. Chem. Phys. 146, 244114 (2017).

${ }^{29}$ K. L. Kounovsky-Shafer, J. P. Hernandez-Ortiz, K. Potamousis, G. Tsvid, M. Place, P. Ravindran, K. Jo, S. Zhou, T. Odijk, J. J. De Pablo et al., "Electrostatic confinement and manipulation of DNA molecules for genome analysis," Proc. Natl. Acad. Sci. U. S. A. 114, 13400-13405 (2017).

${ }^{30} \mathrm{~J}$. Li, X. Jiang, A. Singh, O. G. Heinonen, J. P. Hernández-Ortiz, and J. J. de Pablo, "Structure and dynamics of hydrodynamically interacting finite-size Brownian particles in a spherical cavity: Spheres and cylinders," J. Chem. Phys. (in press).

${ }^{31}$ V. K. Kodali, W. Roos, J. P. Spatz, and J. E. Curtis, "Cell-assisted assembly of colloidal crystallites," Soft Matter 3, 337-348 (2007).

${ }^{32} \mathrm{P}$. Kolhar and S. Mitragotri, "Polymer microparticles exhibit size and shape dependent accumulation around the nucleus after endocytosis," Adv. Funct. Mater. 22, 3759-3764 (2012).

${ }^{33}$ S. B. Savage and C. K. K. Lun, "Particle size segregation in inclined chute flow of dry cohesionless granular solids," J. Fluid Mech. 189, 311-335 (1988).

${ }^{34}$ J. M. N. T. Gray, "Particle segregation in dense granular flows," Annu. Rev. Fluid Mech. 50, 407-433 (2018).

${ }^{35}$ V. Sharma, K. Park, and M. Srinivasarao, "Shape separation of gold nanorods using centrifugation," Proc. Natl. Acad. Sci. U. S. A. 106, 4981-4985 (2009).

${ }^{36}$ R. Caulkin, X. Jia, M. Fairweather, and R. A. Williams, "Geometric aspects of particle segregation," Phys. Rev. E 81, 051302 (2010).

${ }^{37}$ C. Pozrikidis, Boundary Integral and Singularity Methods for Linearized Viscous Flow (Cambridge University Press, Cambridge, 1992).

${ }^{38}$ O. Ladyzhenskaya, The Mathematical Theory of Viscous Incompressible Flow (Gordon and Beach, New York, 1963).

${ }^{39}$ H. Power and L. C. Wrobel, Boundary Integral Methods in Fluid Mechanics (Computational Mechanics Publications, Southampton, 1995).

${ }^{40}$ J. F. Brady and G. Bossis, "Stokesian dynamics," Annu. Rev. Fluid Mech. 20, 111 (1988). 
${ }^{41}$ A. Sierou and J. F. Brady, “Accelerated Stokesian dynamics simulations," J. Fluid Mech. 448, 115-146 (2001).

${ }^{42}$ J. W. Swan and J. F. Brady, "Particle motion between parallel walls: Hydrodynamics and simulation," Phys. Fluids 22, 103301 (2010).

${ }^{43} \mathrm{M}$. Wang and J. F. Brady, "Spectral Ewald acceleration of Stokesian dynamics for polydisperse suspensions," J. Comput. Phys. 306, 443-477 (2016).

${ }^{44}$ A. M. Fiore, F. Balboa Usabiaga, A. Donev, and J. W. Swan, "Rapid sampling of stochastic displacements in Brownian dynamics simulations," J. Chem. Phys. 146, 124116 (2017)

${ }^{45} \mathrm{~A}$. K. Townsend and H. J. Wilson, "Anomalous effect of turning off long-range mobility interactions in Stokesian dynamics," Phys. Fluids 30, 077103 (2018).

${ }^{46}$ A. M. Fiore and J. W. Swan, "Fast Stokesian dynamics," J. Fluid Mech. 878, 544-597 (2019).

${ }^{47}$ T. A. Osswald and J. P. Hernández-Ortiz, Polymer Processing: Modeling and Simulation (Carl Hanser-Verlag, Munich, 2006).

${ }^{48} \mathrm{R}$. Cortez, "The method of regularized Stokeslets," SIAM J. Sci. Comput. 23, 1204 (2001).

${ }^{49}$ A. Kumar and M. D. Graham, "Accelerated boundary integral method for multiphase flow in non-periodic geometries," J. Comput. Phys. 231, 6682-6713 (2012).

${ }^{50}$ C. S. Peskin, "The immersed boundary method," Acta Numerica 11, 479-517 (2002).

${ }^{51}$ P. J. Atzberger, P. R. Kramer, and C. S. Peskin, "A stochastic immersed boundary method for fluid-structure dynamics at microscopic length scales," J. Comput. Phys. 224, 1255-1292 (2007).

${ }^{52}$ B. Kallemov, A. Bhalla, B. Griffith, and A. Donev, "An immersed boundary method for rigid bodies," Commun. Appl. Math. Comput. Sci. 11, 79-141 (2016).

${ }^{53}$ B. Sprinkle, A. Donev, A. P. S. Bhalla, and N. Patankar, "Brownian dynamics of fully confined suspensions of rigid particles without Green's functions," J. Chem. Phys. 150, 164116 (2019).

${ }^{54}$ H. Risken, The Fokker-Planck Equation, 2nd ed. (Springer-Verlag, Berlin, Heidelberg, 1989).

${ }^{55}$ H. C. Öttinger, Stochastic Processes in Polymeric Fluids (Springer-Verlag, Berlin, Heidelberg, 1996).

${ }^{56}$ J. P. Hernández-Ortiz, J. J. de Pablo, and M. D. Graham, "Fast computation of many-particle hydrodynamic and electrostatic interactions in a confined geometry," Phys. Rev. Lett. 98, 140602 (2007).

${ }^{57}$ M. Fixman, "Implicit algorithm for Brownian dynamics of polymers," Macromolecules 19, 1195-1204 (1986).
${ }^{58}$ P. Pranay, S. G. Anekal, J. P. Hernandez-Ortiz, and M. D. Graham, "Pair collisions of fluid-filled elastic capsules in shear flow: Effects of membrane properties and polymer additives," Phys. Fluids 22, 123103 (2010).

${ }^{59}$ P. A. Lebwohl and G. Lasher, "Nematic-liquid-crystal order-A Monte Carlo calculation," Phys. Rev. A 6, 426 (1972).

${ }^{60}$ J. A. Martínez-González, X. Li, M. Sadati, Y. Zhou, R. Zhang, P. F. Nealey, and J. J. de Pablo, "Directed self-assembly of liquid crystalline blue-phases into ideal single-crystals," Nat. Commun. 8, 15854 (2017).

${ }^{61}$ M. D. Carbajal-Tinoco, R. Lopez-Fernandez, and J. L. Arauz-Lara, "Asymmetry in colloidal diffusion near a rigid wall," Phys. Rev. Lett. 99, 138303 (2007).

${ }^{62}$ H. B. Eral, J. M. Oh, D. Van Den Ende, F. Mugele, and M. H. G. Duits, "Anisotropic and hindered diffusion of colloidal particles in a closed cylinder," Langmuir 26, 16722-16729 (2010).

${ }^{63}$ A. E. Cervantes-Martínez, A. Ramírez-Saito, R. Armenta-Calderón, M. A. Ojeda-López, and J. L. Arauz-Lara, "Colloidal diffusion inside a spherical cell," Phys. Rev. E 83, 030402 (2011).

${ }^{64}$ G. L. Hunter, K. V. Edmond, and E. R. Weeks, "Boundary mobility controls glassiness in confined colloidal liquids," Phys. Rev. Lett. 112, 218302 (2014).

${ }^{65} \mathrm{~W}$. Wang and P. Huang, "Anisotropic mobility of particles near the interface of two immiscible liquids," Phys. Fluids 26, 092003 (2014).

${ }^{66} \mathrm{G}$. Nägele, "On the dynamics and structure of charge-stabilized suspensions," Phys. Rep. 272, 215-372 (1996).

${ }^{67}$ J. Balbo, P. Mereghetti, D.-P. Herten, and R. C. Wade, “The shape of protein crowders is a major determinant of protein diffusion," Biophys. J. 104, 1576-1584 (2013).

${ }^{68}$ E. Guazzelli and J. F. Morris, A Physical Introduction to Suspension Dynamics (Cambridge University Press, 2011), Vol. 45.

${ }^{69} \mathrm{~J}$. Mewis and N. J. Wagner, Colloidal Suspension Rheology (Cambridge University Press, 2012).

${ }^{70}$ R. I. Tanner, "Review article: Aspects of non-colloidal suspension rheology," Phys. Fluids 30, 101301 (2018).

${ }^{71}$ A. Singh, R. Mari, M. M. Denn, and J. F. Morris, "A constitutive model for simple shear of dense frictional suspensions," J. Rheol. 62, 457-468 (2018).

${ }^{72}$ A. Singh, S. Pednekar, J. Chun, M. M. Denn, and J. F. Morris, "From yielding to shear jamming in a cohesive frictional suspension," Phys. Rev. Lett. 122, 098004 (2019).

${ }^{73}$ V. Sivadasan, E. Lorenz, A. G. Hoekstra, and D. Bonn, "Shear thickening of dense suspensions: The role of friction,” Phys. Fluids 31, 103103 (2019).

${ }^{74}$ N. M. James, H. Xue, M. Goyal, and H. M. Jaeger, "Controlling shear jamming in dense suspensions via the particle aspect ratio," Soft Matter 15, 3649-3654 (2019). 